\title{
Mens sana in corpore sano: incorporación de la higiene mental en la salud pública en la primera mitad del siglo XX en Colombia*
}

\author{
JaIRo GutTírReZ AvendaÑo
}

Docente investigador de la Universidad Católica Luis Amigó (Colombia). Correo electrónico: jairo.gutierrezav@amigo.edu.co. El autor es doctor en Ciencias Humanas y Sociales de la Universidad Nacional de Colombia. Entre sus publicaciones recientes tenemos: "Historiografía de la locura y de la psiquiatría en Colombia. De los médicos escritores a la perspectiva crítica, 1968 - 2018”, HiSTORelo Vol.10 No. 21 (2018) y "Degeneración, delirios y nervios: etiología en la primera psiquiatría 1893-1912". En: Restrepo Z., Estela; Sánchez B., y Silva C., Gustavo (Eds). ciencias de la vida (T. 1). Bogotá: Universidad Nacional de Colombia, Colección Sesquicentenario, 2017. Entre sus temas de interés están: Fenomenología e Historia de la locura y de la psicopatología.

Recibido: 11 de diciembre de 2017

Aprobado: 21 de mayo de 2018

Modificado: 30 de mayo de 2018

Artículo de investigación científica

DOI: http://dx.doi.org/10.15648/hc.34.2019.3

* $\quad$ Este artículo forma parte de la tesis doctoral: Jairo Gutiérrez, "Locura y sociedad. Alienismo tardío, psicopatología e higiene mental en la modernidad colombiana, 1870-1968", (tesis de Doctor en ciencias Humanas y Sociales, Universidad Nacional de Colombia, 2017), financiado por la Universidad Católica Luis Amigó (Colombia).

Esta publicación está bajo una licencia Creative Commons Reconocimiento-NoComercial 4.0 


\title{
Mens sana in corpore sano: incorporación de la higiene mental en la salud pública en la primera mitad del siglo XX en Colombia
}

\section{Resumen}

El presente artículo tiene por objetivo comprender las apropiaciones y transposiciones científicas entre higiene mental como solución técnica y eugenesia como ideología, en el surgimiento del concepto de salud mental a mediados del siglo XX en Colombia. Metodología: inscrita en la corriente de historia social de la ciencia, se realizó una investigación documental, mediante heurística de archivo y hermenéutica. Conclusiones: el predominio de la propensión hereditaria como determinante de la degeneración, por sobre los factores ambientales, tuvo transposiciones discursivas y prácticas en la eugenesia, extendida a tres campos: la higiene (física, mental y moral), la puericultura o cuidado materno-infantil, y la homicultura para "labrar el cuerpo y cosechar al hombre". La higiene mental constituyó una solución técnica para prevenir y remediar la decadencia de la raza a la que se atribuía el atraso civilizatorio en que se encontraba sumido el país, trasfondo histórico que caracterizó el surgimiento de la incorporación del cuidado psíquico en la agenda de la salud pública en Colombia y Latinoamérica.

Palabras clave: Higiene mental, salud mental, eugenesia, degeneración, locura.

\section{Mens sana in corpore sano: incorporation of mental hygiene into public health during the first half of the 20th century in Colombia}

\begin{abstract}
The present article aims to understand the appropriations and scientific transpositions between mental hygiene as a technical solution and eugenics as ideology, in the emergence of the concept of mental health in the middle of the 20th century in Colombia. Methodology: inscribed in the current of social history of science, a documentary research was carried out, by means of heuristics of archives and hermeneutics. Conclusions: the predominance of hereditary propensity as a determinant of degeneration, over environmental factors, had discursive and practical transpositions in eugenics, extended to three fields: hygiene (physical, mental and moral), puericulture or maternal-child care and homiculture to "work the body and reap the man". Mental hygiene was a technical solution to prevent and remedy the decline of the race that was attributed to the civilization backlog in which the country was plunged, a historical background that characterized the emergence of the incorporation of psychic care in the public health agenda in Colombia and Latin America.
\end{abstract}

Keywords: Mental hygiene, mental health, eugenics, degeneration, insanity. 


\section{Mens sana in corpore sano: incorporação da higiene mental na saúde pública durante a primeira metade do século XX na Colômbia}

\section{Resumo}

No presente artigo tem como objetivo compreender as apropriaçóes e transposiçóes científicas entre higiene mental como solução técnica e eugenia como ideologia, no surgimento do conceito de saúde mental em meados do século XX na Colômbia. Metodologia: inscrita na corrente da história social da ciência, foi realizada uma pesquisa documental através de heurísticas de arquivamento e hermenêutica. Conclusóes: o predomínio da propensão hereditária como determinante da degeneração, por cima dos fatores ambientais, Teve transposições discursivas e práticas na eugenesia, difundida em três campos: a higiene (física, mental e moral), a puericultura ou cuidado materno-infantil e a homicultura para "lavrar o corpo e colher ao homem", a higiene mental representou uma solução técnica para evitar e resolver a decadência da raça à qual se atribuía o atraso civilizacional em que se encontrava mergulhado o país, cenário histórico que caracterizou o surgir da incorporação do cuidado psíquico no calendário da saúde pública na Colômbia e América Latina.

Palavras-chave: higiene mental, saúde mental, eugenia, degeneração, loucura.

\section{Mens sana in corpore sano: incorporation de l'hygiène mentale dans la santé publique dans la première moitié du XXème siècle en Colombie}

\section{Résumé}

Cet article a pour objectif de comprendre les appropriations et les transpositions scientifiques entre hygiène mentale comme solution technique et eugénisme comme idéologie, dans la naissance du concept de santé mentale au milieu du XXème siècle en Colombie. Méthodologie : inscrite dans le courant d'histoire sociale de la science, on a réalisé une recherche documentaire, à travers l'heuristique des archives et de l'herméneutique. Conclusions : la prédominance de la propension héréditaire comme déterminant de la dégénérescence, au-dessus de facteurs environnementaux, a eu de transpositions discursives et pratiques dans l'eugénisme, répandue à trois domaines: l'hygiène (physique, mentale et morale), la puériculture ou le soin materne-infantile, et l'homiculture pour «tailler le corps et cultiver l'homme», L'hygiène mentale a constitué une solution technique pour prévenir et remédier la décadence de la race à laquelle on attribuait le retard civilisateur dans lequel le pays se trouvait plongé, arrière- plan historique qui a caractérisé la naissance de l'incorporation du soin psychique dans l'agenda de santé publique en Colombie et en Amérique Latine.

Mots clés: Hygiène mentale, santé mentale, l'eugénisme, la dégénérescence, la folie. 


\section{Movimiento de higiene Mental y Despliegue Del CONCEPTO DE SALUD MENTAL}

Más allá de la Mens sana in corpore sano, virtud perenne para una "vida tranquila" ${ }^{1}$, ejercitada por diferentes tradiciones culturales de Occidente, una versión moderna se asumió como "higiene mental" (HM), cuyo primer término, usualmente atribuido a la salud física, la salubridad y prevención de la enfermedad, se asoció con una nueva tecnología del cuidado de sí para el gobierno de las pasiones, el control emocional y la salud psíquica. Este sentido se identificó con el carácter del primer movimiento que dio origen a las ligas de higiene mental en Estados Unidos.

La historia del Mental Hygiene Movement, de acuerdo con Gerald Grob ${ }^{2}$, constituye "un ejemplo de cómo los cambios estructurales pueden llenar viejos conceptos con nuevos significados, y cómo una organización y un movimiento pueden influir en la vida de grupos dependientes como los enfermos mentales"3.

El Mental Hygiene Movement surgió en 1908 por iniciativa del médico Clifford Beers, quien padecía depresión y paranoia y relató su experiencia como paciente en una institución psiquiátrica, en su libro $A$ mind that found itself. Esta obra propugnó por revelar y divulgar la necesidad de trascender la prevención y la intervención de la enfermedad psíquica e innovar en su tratamiento mediante la promoción comunitaria de la "salud mental”.

Este propósito exigía la implementación de reformas y medidas administrativas que, a la postre, hicieron que esta nueva concepción (y su concep-

1 Por ejemplo, en Colombia, uno de los primeros discursos de higiene escolar que asumió dicha consigna — sin el referente del Movimiento de Higiene Mental norteamericano-, puede atribuirse al publicado por Carlos De Greiff, "Mens sana in corpore sano", Anales de la Academia de Medicina de Medellín, Ańo XII Tomo 12, $\mathrm{N}^{\circ} 1$ y 2, agosto 30 (1903).

2 Profesor emérito de Historia de la Medicina de Rutgers, The State University of New Jersey, Institute for Health, Health Care Policy.

3 Gerald Grob, "The Mental Hygiene Movement", en Mental Illnes and American Society, 1875-1940 (cap. 6) (Princeton: Princeton University Press, 1987), 144-178, traducción: María Laura Moukarzel, revisión: Hernán Scholten y Agustín Kripper. 
to relacionado "higiene mental") fuera incorporada como programa en las políticas públicas de salud 4 . De todas maneras, el concepto de higiene mental permaneció por varias décadas sin ser adoptado en el uso técnico común y oficial hasta que, en la década de 1950, como se verá, fue definido por el Comité de Expertos de Higiene Mental de la Organización Mundial de la Salud.

No obstante, la referencia a la HM no era nueva, puesto que en 1843 la había utilizado en Estados Unidos el médico William Sweetser ${ }^{5}$ en un artículo titulado igual que dicho concepto; asimismo, en 1857, fue incluida en Inglaterra por John Hawkes como parte de un "Programa para promover la reforma sanitaria mental"6; luego, en 1859, George Cook ${ }^{7}$ la refiere en dos artículos sobre la protección de la salud y la profilaxis de la enfermedad psíquica.

Interesa este antecedente porque en el caso de Colombia, se empleaba el concepto de HM, como parte de la higiene social, desde finales del siglo XIX, antes de la aparición del Mental Hygiene Movement. También fue utilizado a comienzos del siglo XX, sin que existiera en Colombia una sociedad o liga de HM, como se constata en el debate sobre la degeneración de la raza, en médicos como Jiménez López, López de Mesa, Bejarano y otros (1920), quienes lo asociaron a la terapéutica de la medicina social, así como a la eugenesia, como en el caso de Eduardo Vasco, de quien se comentará más adelante.

De acuerdo con Rosen, la historia de la medicina social es, en gran parte, la de la política y de la acción social en relación con los problemas de la salud $^{8}$, como lo exponen Dagfal, para el contexto de Argentina ${ }^{9}$ y Ríos, para

4 Paul Lemkau, Higiene mental (México: Fondo de Cultura Económica, 1963), 17.

5 William Sweetser, como se citó en George Rosen, Locura y sociedad, sociología histórica de la enfermedad mental (Madrid: Alianza, 1974), 329.

6 John Hawkes, como se citó en George Rosen, Locura y sociedad, 329.

7 George Cook, como se citó en George Rosen, Locura y sociedad, 329.

8 George Rosen, De la policía médica a la medicina social. Ensayos sobre la historia de la atención en salud (México: Siglo XXI, 2005), 9.

9 Alejandro Dagfal, "El pasaje de la higiene mental a la salud mental en la Argentina, 1920-1960. El caso de Enrique Pichon-Rivière”, Trashumante. Rev. Amer. Hit. Soc. No.5 (2015): 10-36. 
el de México. No obstante, antes que reducir la eugenesia a un proyecto ideológico estatal y la higiene mental a la ampliación psiquiátrica de dicha gubernamentalidad, es crucial comprender que ambas tuvieron en común "las condiciones generadas por la salud pública en la década de 1930 con la medicina social como referente central" ${ }^{10}$, que les permitieron su intervención y legitimización sociopolítica.

Si bien Ríos plantea, para el caso mexicano, que la diferencia entre eugenesia e higiene mental "consistió en el distanciamiento de la higiene mental con el paradigma degeneracionista y con los discursos organicistas para comprender la enfermedad mental (...) a diferencia de la eugenesia cuyo referente estaba enclavado en la herencia" ${ }^{11}$, en el contexto colombiano dicho distanciamiento se estableció con la circunscripción de la eugenesia a la puericultura, más cercana al discurso y a la práctica de la HM, aunque esta última mantuvo rezagos del postulado de la determinación y predisposición hereditaria hasta mediados del siglo XX, como se verá más adelante.

Las sociedades de higiene mental fueron escalando hacia la conformación de comités nacionales con una representación de psiquiatras, médicos de otras especialidades, biólogos, pedagogos, religiosos, empresarios, sociedades de filantropía, representantes de la sociedad civil, entre otros, de un gran número de regiones, hasta extenderse a países de Europa, África, Asia y Latinoamérica.

En 1930 se realizó el First International Congress on Mental Hygiene, celebrado en Washington bajo la dirección de Beers ${ }^{12}$. Asistieron 3000 representantes de 41 países, del que no se halló registro de algún delegado

10 Andrés Ríos, ¿Cómo prevenir la locura? Psiquiatría e higiene mental en México, 1934-1950 (México D.F.: UNAM - Siglo XXI, 2016), 154.

11 Andrés Ríos, ¿Cómo prevenir la locura?, 15.

12 Previo al de Washington, en Francia la Ligue d'Hygiène et Prophylaxis Mentales, en cabeza del psiquiatra y periodista Edouard Toulouse, organizó en Paris el Congrès d' Hygiène Mentale, en junio de 1922, con una representación de 22 países, fue en su origen un intento de celebrar el primer congreso internacional de higiene mental; sin embargo, "Estados Unidos se opuso y reivindicó el derecho a celebrar el primer evento de este tipo por ser los creadores del movimiento de Higiene Mental. El congreso celebrado en París quedó reducido a una reunión europea". Ver: Ricardo Campos, "De la higiene del aislamiento a la higiene de la libertad. La reforma de la institución manicomial en Francia (1860-1940)", Frenia, Vol. 1, No. 1, (2001): 37-64. 
colombiano. En este evento se dio el voto unánime para que la higiene mental se incluyera en los planes de estudio de la carrera de medicina e influyó en la formación de la psicología experimental y la implementación de la psicotecnia, antecedentes que posteriormente dieron origen a los primeros institutos y programas profesionales de psicología general.

Los alcances del movimiento de HM y su congreso internacional sentaron las bases para la conformación del primer Comité de Expertos de HM de la Organización Mundial de la Salud, organizado en Ginebra en 1949, el cual estuvo integrado por médicos directores de hospitales psiquiátricos de China, Checoeslovaquia, Estados Unidos, India y Brasil.

Este Comité estableció dos principios de su accionar. El primero fue el de la inclusión oficial de la HM en los servicios de salud pública. Según este se propuso la creación de una especialidad en la materia, así como la divulgación de normas de vida en colectividad que se impartieran con igual importancia a la que se daba a la salud física ${ }^{13}$.

El segundo principio buscó concentrar los esfuerzos de la psiquiatría terapéutica y profiláctica en las fases de la infancia, dado que resultaba más efectiva para la prevención temprana de la psicopatología y la criminalidad. En ese mismo campo propuso que a los maestros de escuela se les debía adiestrar en el diagnóstico o reconocimiento de los problemas graves de personalidad y disciplina en su estado incipiente, antes que en su tratamiento, para lo que no estarían facultados; también se propuso que a los padres de familia se les instruyera sobre pautas de crianza de los hijos para evitar la inclinación a transgredir las normas ${ }^{14}$.

El Comité planteó la urgencia de cambiar el modelo manicomial de largos periodos de hospitalización como se administraba en Europa Occidental y Estados Unidos. Hacía notar el contraste con otros países como China e India, en los que el cuidado familiar parecía ser un método más

13 Organización Mundial de la Salud, Informe de la Primera reunión del Comité de Expertos en Higiene Mental, Ginebra, Oficina Sanitaria Panamericana, Washington, Serie de Informes Técnicos, 1949, No. 31, 7 .

14 OMS, Informe de la primera reunión del Comité de Expertos, 26-27. 
satisfactorio $^{15}$. Se destaca que, en Colombia, los cuidados domiciliares se propusieron claramente en las décadas de 1950 y 1960, en los modernos hospitales mentales de Antioquia y Valle del Cauca, los cuales entraron en funcionamiento casi a la par, entre 1959 y 1960.

Asimismo, recomendó la terapéutica de la psiquiatría dinámica que implicaba la aplicación progresiva y completa de las ciencias biológicas, psicológicas, sociales y antropológicas al estudio de la etiología, patología y tratamiento de los trastornos mentales. No obstante, de acuerdo con Juan D. Lopera, el concepto técnico de salud mental emitido por la OMS respondía más a fundamentos políticos e ideológicos que científicos, puesto que este no fue resultado de un desarrollo "intracientífico", es decir, no surgió de una relación directa con las investigaciones científicas en ese campo ${ }^{16}$, sino con preceptos del saber comportarse en comunidad.

En efecto, una de las críticas es su incompatibilidad con los postulados del Mental Hygiene Movement, al que suele atribuirse su origen. En efecto, desde cuando se creó la OMS, en 1946, en su Acta de constitución, se contempló que una de sus funciones era "fomentar las actividades en el campo de la salud mental, especialmente aquellas que afectan la armonía en las relaciones humanas".

Sin embargo, según lo señala Lopera, en la versión española del Acta de constitución se tradujo mental health por "higiene mental"17. Este cambio muestra la confusión entre ambos términos, como lo sugiere el informe de la Segunda reunión del Comité de Expertos realizada en 1950, porque la HM "se emplea en sentido eufemístico para designar el tratamiento psiquiátrico temprano, presumiblemente para evitar el estigma que aún se le imputa con frecuencia a los trastornos psicóticos establecidos y, a través de estos, a la palabra 'psiquiatría"'18.

15 OMS, Informe de la primera reunión del Comité de Expertos, 8.

16 Juan D. Lopera, "Una historia del concepto técnico de salud mental: entre ciencia e ideología”, en Salud y Salud Pública. Aproximaciones históricas y epistemológicas, eds. Álvaro Casas y Jana Congote (Facultad Nacional de Salud Pública, U. de A. - Hombre Nuevo Editores, 2013), 35-36.

17 Lopera, Una historia del concepto técnico de salud mental, 45.

18 Organización Mundial de la Salud, Informe de la segunda reunión del Comité de Expertos en Higie- 
Por lo tanto, el Comité definió que la primera es un procedimiento que contribuye a la segunda, es decir, la higiene mental es el desarrollo de técnicas y actividades que promueven la salud mental. En ese sentido, se buscó superar la ambigüedad y establecer un proceso para alcanzar el ideal de bienestar, felicidad, equilibrio, etc., que, a su vez, debía llevar a la "armonía en las relaciones humanas", contemplada en el concepto de la OMS. Resulta evidente que este se refiere a un régimen tranquilizante de la vida o que sigue la consigna "practicar la higiene es saber vivir", también adoptada en los tratados de higiene en Colombia, analizados más adelante.

Según Grob, una de las críticas al movimiento de HM es que -a diferencia del ideal de la profilaxis psiquiátrica de finales del siglo XIX, priorizada en la población asilada- al descentralizar su función intramuros hacia una acción más preventiva que terapéutica, desfavoreció una mayor atención a los que ya se encontraban en condición de enfermos mentales, limitados en su autonomía para defender sus intereses ${ }^{19}$.

Asimismo, de acuerdo con Paul Lemkau ${ }^{20}$, este cambio de postura, también generó un distanciamiento de los psiquiatras con respecto a la población normal, al igual que con la profesión médica en general ${ }^{21}$. Más allá de la higiene mental extendida al ámbito de la vida privada, de manera paradójica, esta resultó propicia para mejorar la reputación de la profesión psiquiátrica, motivación que tuvo más empeño que el de aliviar el sufrimiento psíquico de los pacientes.

Otro cuestionamiento, según Ríos en el caso de México, es que "cuando la higiene mental fue reemplazada por el modelo de salud mental, las ligas e individuos perdieron el liderazgo que ostentaban en el modelo anterior,

ne Mental, Ginebra, Oficina Sanitaria Panamericana, Washington, Serie de Informes Técnicos, 1950, No. 31, 2-3.

19 Gerald Grob, The Mental Hygiene Movement, 144.

20 Psiquiatra fundador del School's Department of Mental Hygiene, director del Baltimore's Eastern Health District Project. Reconocido gestor de la salud mental comunitaria y de su inclusión en la política pública de salud en Estados Unidos, su obra constituyó un referente en varios países como Japón, Yugoslavia e Italia, así como en Latinoamérica.

21 Paul Lemkau, Higiene Mental, 17. 
debido a que las políticas e instituciones de salud mental debían estar administradas por el Estado y no por la sociedad civil"22. En Colombia, por el contrario, no existió un movimiento social en pro de la salud mental, sino que fue producto de la gestión en instancias administrativas y ministeriales al principio fallidas, a finales de la década del cincuenta y puestas en marcha en los ańos sesenta, como se verá en el último apartado.

A la Segunda Reunión de la Asociación Psiquiátrica de América Latina (APAL) y al IV Congreso Mundial de Salud Mental, realizados conjuntamente en Ciudad de México, en 1951, asistieron delegados de Argentina, Colombia, Costa Rica, Cuba, Chile, Ecuador, Guatemala, Honduras, Panamá, Perú, República Dominicana, Venezuela y México. En este evento sobresale un mayor uso del concepto de higiene mental, que el de mental bealth, recién oficializado por la OMS el año anterior.

Se destaca que el presidente de la primera sesión de esta segunda reunión de la APAL fue el médico colombiano Eduardo Vasco Gutiérrez, precursor de la psiquiatría infantil y de la HM en el país, sobre el que se comentará más adelante. Según el informe del secretario Ramón Alcerro, delegado de Honduras, se destaca la intervención de A. Repond ${ }^{23}$, sobre el tema "Esfuerzos de la comunidad en la higiene mental", en el que comentó que "los conocimientos, los métodos y la aplicación de la higiene mental están aún en sus comienzos, pero lo poco que sabemos nos da a todos aquellos dedicados a su difusión, la seguridad de que al fin será uno de los más importantes factores en el desarrollo y mejoramiento del género humano" ${ }^{24}$.

Esta percepción era similar a la que Vasco enunciara en su libro Breviario de la madre (1934). Ahí afirmó que la HM era casi desconocida en Colombia y, por tanto, no se habían adelantado esfuerzos científicos para su formación en el campo de la salud y fomentarla entre la población ${ }^{25}$.

22 Andrés Ríos, ¿Cómo prevenir la locura?, 202.

23 Director de la Maison de la Santé de Malévoz, en Suiza. Miembro del Comité de Expertos en Salud Mental de la OMS, fundado en Ginebra en 1949, sobre el que se comentó en el apartado anterior.

10024 Ramón Alcerro, "Informe acerca de las actividades de la Segunda Reunión de la Asociación Psiquiátrica de América Latina y del IV Congreso Mundial de Salud Mental”, Revista Médica Hondureña, No. 20, (1952): 80-88.

25 Eduardo Vasco, Breviario de la madre (Medellín: Bedout, 1934), 8-11. Biblioteca Central U. de A., 
En la reunión de 1951, Erich Fromm disertó sobre la "contribución de las ciencias sociales a la Higiene Mental". Sostuvo que "el psiquiatra y el psicólogo deben armonizar su trabajo con el del economista, el sociólogo, el antropólogo y el político científico y también con el del estudiante de filosofía y ética, para poder presentar aún las más tentativas sugestiones para la organización de una sociedad más encaminada al desenvolvimiento humano y a la producción y armonía; esto es, a la salud mental"26.

Uno de los temas del evento estuvo dedicado a "La Salud Mental de los niños". En este fueron ponentes P. Lemkau, A.M. Norvig, y T. Reca. Esta última, en su intervención enfatizó que "no puede concebirse una obra de higiene mental en América Latina separada de una acción tendiente al mejoramiento de las condiciones de vida, vivienda y salud de la familia latinoamericana" ${ }^{27}$.

Por su parte, la doctora Norvig planteó que "esta responsabilidad descansa principalmente en la administración. Para el logro de un ambiente social saludable en las aulas, los psiquiatras y psicólogos deben aliarse con los maestros, para exigir una política; clara y democrática dentro del programa escolar" ${ }^{\prime 28}$.

Se destaca que en las intervenciones sobre HM tuvo relevancia el modelo de la Child Guidance Clinics (CGC), creado por el National Comittee for Mental Hygiene desde la década de 1920 y que seguía vigente en la década de 1950. A este modelo del CGC se le atribuye el origen de la psiquiatría infantil, así como iniciativas de tratamiento y prevención de la delincuencia juvenil, trastornos de la conducta y de la personalidad para la población de 3 a 17 años de edad, de los niños que no fueran "retrasados mentales" 29 . Se destaca que la era de la CGC representó la primera vez que la salud mental de los niños se consideró desde un enfoque independiente.

Sala de Patrimonio Documental.

26 Ramón Alcerro, Informa acerca de la actividades, 80-88.

27 Ramón Alcerro, Informa acerca de la actividades, 80-88.

28 Ramón Alcerro, Informa acerca de la actividades, 80-88.

29 Victor Anderson, "The Organization and Operation of Child Guidance Clinics", The Public Health Journal, Vol. 16, No. 8, August (1925): 371-378. Recuperado 29/05/2015. Disponible en: https:// www.jstor.org/stable/41973349?seq=1\#page_scan_tab_contents 
En efecto, según Albord,

[...] la importancia de los médicos escolares capacitados para reconocer un desequilibrio emocional, en su período inicial, son de suma importancia para iniciar el tratamiento psiquiátrico antes que la desadaptación se vuelva seria o tenga profundas raíces. Para hacer frente a esta necesidad el servicio de guía infantil debe ser incorporado en todos los servicios médicos escolares ${ }^{30}$.

De acuerdo con Rosen, la CGC promovió la creación de la American Orthopsychiatric Asociation ${ }^{31}$. Como se verá en adelante, en Colombia esta corriente se enfocó en la reforma "psico-médico-pedagógica" del régimen correccional de las casas de menores y en la creación de centros de orientación de la infancia.

\section{IRRUPCiÓN DE LO MENTAL EN LOS CAMPOS DE LA HIGIENE Y LA ME- DICINA SOCIAL EN COLOMBIA}

Si bien, en la primera mitad del siglo XX, se tendía al reduccionismo de asumir que las intensidades psíquicas de la modernidad eran causantes de los desórdenes mentales, de acuerdo con Lopera, más bien se trató de un contexto histórico de "racionalización del mundo de la vida", "movilización general", "aceleración" o "inquietud" en el que se hicieron visibles desórdenes psíquicos que llamaron la atención de la opinión pública y, con el surgimiento de la psiquiatría desde el siglo XIX, entraron en el dominio de la clasificación de las enfermedades mentales ${ }^{32}$.

Los preceptos de la mente sana en cuerpo sano y la higiene como sabiduría práctica fueron comunes entre los médicos. Es el caso de Carlos De Greiff, quien publicó artículos sobre el tema desde 1889 en los Anales de la Academia de Medicina de Medellín, los cuales fueron integrados en su libro de Conferencias de Higiene en las Escuelas de Medellín, en 1905, dirigidas a la Instrucción Pública y extendidas a otros ámbitos de la urbanidad en los

30 Ramón Alcerro, Informa acerca de la actividades, 80-88.

31 George Rosen, Locura y sociedad, 328.

32 Juan D. Lopera, "Sabiduría práctica y salud psíquica" (tesis de Doctorado en Ciencias Sociales, Universidad de Antioquia, Medellín, 2014), (Bogotá: Editorial San Pablo - Fondo Editorial Universidad Eafit, 2016), 27. 
que se incluyeron nociones de higiene social, moral, del matrimonio, entre otras materias; así, utilizó el siguiente silogismo:

"La higiene está íntimamente ligada a la moral y la urbanidad: casi todas las reglas de urbanidad que nos enseña Carreño son preceptos higiénicos; el que quebranta la moral, atenta casi siempre contra la salud, y por eso peca contra la higiene; el que no observa los preceptos higiénicos atenta contra la salud y por consiguiente quebranta la moral"33.

Las conferencias de Carlos De Greiff, distribuidas en 14 sesiones, divulgaron una serie de temáticas típicas de la higiene de la época, tales como los buenos y malos hábitos, el régimen alimenticio; ergonomía escolar y "esfuerzos de acomodación", fatiga o recargo escolar, hidratación y purificación del agua y baño diario, adecuada circulación del aire en relación con la prevención de la tuberculosis; iluminación del espacio, vestido acorde con el clima y la moral, educación física, limpieza del hogar, cuidado de los órganos de los sentidos, higiene del alma adaptada a la moral en relación con las medidas anteriores que influían en la salud y la conducta ${ }^{34}$.

Esta última se extendía a la manera de la episteme de la semejanza desde la cual, según Foucault, se interpretaba por analogía, conveniencia, simpatía y emulación ${ }^{35}$; por ejemplo, la propia consigna de una mente sana en cuerpo sano suponía que la exterioridad era un reflejo del interior; según los hábitos se forjaba la personalidad; asimismo, determinado ambiente sano o malsano suponía el temperamento; la iluminación influía en la irritación, no solo de los ojos, sino del carácter; la indigestión mental se explicaba según la asimilación alimentaria, etc. Similitudes que, como se vio, fueron características del determinismo geográfico y climático en la retórica higienista del degeneracionismo.

De Greiff se basó en los postulados de higiene social del médico Ray Ly-

33 Carlos De Greiff, Segunda conferencia sobre higiene, dictada en la Agrupación Central número $1^{\circ}$, Instrucción Pública Antioqueña, Medellín, 1, (5-6), julio, 1905, 195.

34 Carlos De Greiff, Conferencias de Higiene en las Escuelas de Medellín (Medellín: Tipografía del Comercio, 1905), Sala Patrimonial Universidad Eafit, Medellín.

35 Michel Foucault, Las palabras y las cosas (México: Siglo XXI, 1982). 
man, presidente de la American Social Hygiene Association en la década de 1920. Lyman consideraba que para lograr el propósito de la eugenesia, mediante programas higiénico-educativos, era crucial el mejoramiento de la "eficacia del cerebro, como instrumento material del espíritu" 36 . Sobre este campo de acción, Lyman consideraba:

"De no gozar de salud suficiente la raza, no hay progreso definitivo. En esa marcha hacia la meta de la salud y la dicha, han participado las viviendas salubres, la higienización del agua y de la leche, la mejor asistencia de las criaturas y de las madres, y la mayor atención a la salud en la infancia, y, es más, en toda la vida" ${ }^{37}$.

Las conferencias de De Greiff, así como los discursos de otros higienistas comentados más adelante, constituyen un referente para la historia de la cultura física en el ámbito educativo, quienes, basados en la tradición de la fisiología mecanicista de finales del siglo XIX, enfatizaron en el cuidado del cuerpo mediante el ejercicio como dispositivo higiénico indispensable para contrarrestar el supuesto retraso en que estaba sumida la nación ${ }^{38}$.

Por ejemplo, para De Greiff, "la salud y el vigor físico ejercen notable influencia sobre el cerebro y los sentimientos [...] El raquitismo físico corre parejo con el raquitismo moral y á veces con el raquitismo intelectual, pues las leyes que rigen lo moral y lo intelectual, son las mismas que rigen la materia" 39 .

Por lo tanto, consideraba que el descuido de la actividad física era causante de "esas neurosis que conducen á los hombres a la morfinomanía, el etilismo y á todas las demás toxicomanías. Todo eso hace perder la aptitud para el trabajo, deprime el carácter, rebaja la dignidad humana y termina en la degeneración de la raza. ¡Ojalá tuviéramos menos intelectualidad y gentes más fuertes y más vigorosas!”0. Esta concepción tenía un doble propósito: inculcar un régimen mental para crear conciencia de cuidado del cuerpo.

36 Carlos De Greiff, Mens sana in corpore sano, 49.

37 Ray Lyman, "Un mensaje de higiene social”, Jour. Sor. Hyg., 127, marzo (1937). Reproducido por la Oficina Sanitaria Panamericana, (1937): 1150.

38 Zandra Pedraza, En cuerpo y alma. Visiones del progreso y de la felicidad. Educación, cuerpo y orden social en Colombia (1830-1990) (Bogotá: Universidad de los Andes, 2011), 221.

39 Carlos De Greiff, "Decadencia mental”, Anales de la Academia de Medellín, Vol. 11 No. 1y2, agosto (1899): 48-52.

40 Carlos De Greiff, Mens sana in corpore sano, 50. 
De acuerdo con José Silva, sobre la relación entre cuerpo, espacio y subjetividad en el Manicomio Departamental de Antioquia (1875-1930), la hibridación de los ámbitos higiénicos, físico-moral-mentales, correspondía a tres órdenes en los que la medicina englobaba lo humano a principios del siglo $\mathrm{XX}^{41}$.

En 1913 se realizó en Medellín el Segundo Congreso Médico de Colombia, en el que se programó una "Sección de higiene", de la que se destacan tres conferencias enfocadas en el ámbito educativo: la de José Ignacio Vernaza, la de Alfonso Castro y la de Alberto Borda. El primero se graduó en Medicina de la Universidad Nacional de Colombia, en 1912, con la tesis "Higiene escolar", de la que fue jurado Pablo García Medina, quien ponderó que el trabajo tenía "claramente expuestas las doctrinas y leyes que la higiene preconiza para contrarrestar la influencia depresora del trabajo intelectual y para dar a los nińos y jóvenes educandos el vigor y la salud que han menester en la lucha de la vida y para asegurar el porvenir de la raza" ${ }^{\text {" }}$.

Vernaza planteaba que la educación inicial era el medio crucial de la labor de la higiene, puesto que "en el alma de los niños es en donde debemos modelarla, pues sus cerebros son una célula en formación en donde quedarán vibrando, por siempre, las primeras impresiones que reciban"43.

Asimismo, se enfocaba en la dosificación del trabajo corporal e intelectual en el espacio de la clase para reducir el recargo o surmenage, causante de "decadencia psico-física" que, según se vio en el discurso degeneracionista, se manifestaba en neurastenias, fatigas cerebrales y diferentes dolencias, como lo refirieron también De Greiff ${ }^{44}$, Jiménez ${ }^{45}$, López de Mesa ${ }^{46}$, Cas$\operatorname{tro}^{47}, V_{a s c o}{ }^{48}$, entre otros médicos.

41 José A. Silva, "Espacio, cuerpo y Subjetividad en el Manicomio Departamental de Antioquia: 18751930" (tesis de Maestría en Historia, Universidad Nacional de Colombia, 2012), 137.

42 José Vernaza, "Higiene escolar" (tesis, Facultad de Medicina y Ciencias Naturales, Universidad Nacional de Colombia, Bogotá: Editores Arboleda y Valencia, 1912), 8.

43 José Vernaza, Higiene escolar, 10.

44 Carlos De Greiff, Segunda conferencia sobre higiene, 195.

45 Miguel Jiménez, Luis López de Mesa, Jorge Bejarano, et al., Los problemas de la raza en Colombia segundo volumen (Bogotá: Biblioteca de Cultura, 1920), 32.

46 Luis López de Mesa, "Higiene Mental. Plan quinquenal del Ministerio de Educación Nacional", Anales Neuropsiquiátricos, Vol. 1 (1957): 17. Escritos sobre medicina (Serie documental), folios 1-6, Archivos personales. Patrimonio documental U. de A.

47 Alfonso Castro, Degeneración colombiana (Medellín: Lit. e Imp. J.L. Arango, 1920), 14.

48 Eduardo Vasco, Temas de higiene mental, educación y eugenesia (Medellín: Bedout, 1948), 14. 
El médico escritor Alfonso Castro presentó la ponencia "La higiene de las escuelas" en el congreso médico de 1913. Sostenía que la higiene era la base para combatir la degeneración de la raza en Colombia, solución técnica en la que también coincidieron los médicos Miguel Jiménez López (psiquiatra), Luis López de Mesa (psicólogo), y los higienistas Jorge Bejarano y Emilio Robledo, entre otros, durante el consabido debate sobre la decadencia de la raza.

El ingeniero sanitario Alberto Borda Tanco presentó la conferencia "Higiene escolar y edificios para escuelas", en la que definió la higiene como la ciencia que fija reglas "aplicables a todas las funciones tanto psíquicas como físicas, aplicables a todos los momentos de la vida, estas reglas conducen como último fin al perfeccionamiento del individuo y a su mayor utilidad para la sociedad" 49 . De estos se destaca el surgimiento del médico escolar, la educación física, así como una inscripción en el discurso degeneracionista. Interesa en especial la diferencia entre decadencia intelectual y psíquica, que será comentada más adelante.

El mismo año del referido Segundo Congreso Médico en Colombia, se llevó a cabo el Quinto Congreso Médico Latinoamericano, celebrado en Lima en 1913, en el que participó una delegación de Colombia. Entre las conclusiones, se recomendó la enseñanza de la higiene sexual, la campaña antialcohólica y la enseñanza de la puericultura en las escuelas latinoamericanas, y establecer medidas sanitarias para "la protección de la infancia moralmente abandonada, como medio de combatir la delincuencia, el alcoholismo, la tuberculosis y otras dolencias físicas y morales que estorban el mejoramiento y perfeccionamiento de la raza" ${ }^{50}$.

\section{Transposiciones entre eugenesia, puericultura e higiene MENTAL}

Según los discursos médicos en publicaciones y congresos científicos de la lombia (Bogotá: Escuela Tipográfica Salesiana, 1913), 18.

50 Luis Cuervo, "Quinto Congreso Médico Latinoamericano, Informe de la Delegación Colombiana", Revista Médica de Bogotá, Año 32, No. 380, febrero (1914): 75. 
primera mitad del siglo XX en Colombia, la eugenesia se asimiló a la puericultura, tomando la parte por el todo, así como aparecen emparentadas en la terapéutica de la degeneración y en el caso de menores anormales apropiada en 1921 por Tomás Cadavid y David Velásquez, quienes se refieren a la eugénica del obstetra Adolphe Pinard, precursor del cuidado perinatal o preconcepcional moderno que buscaba cuidar del niño a través de la madre ${ }^{51}$.

No obstante, más allá del discurso, no era factible en la práctica, como ocurría en el Hospicio de Bogotá, en 1922, donde Pablo Llinás y Manuel Ortiz informaron que "no hemos creído que la Asamblea pretendiera establecer una intensa obra de Eugénica cuyo primer peldaño quedara señalado en las Salas-cunas; ni una mediana Eugenésica limitada, según el concepto de Pinard; pero tampoco una Puericultura práctica completa, sino apenas el consumo modesto e indeficiente de aquellas medidas protectoras de la vida de los recién nacidos menesterosos" 52 .

La Ley 12 de 1926 "sobre enseñanza de la higiene, saneamiento de los puertos marítimos, fluviales y terrestres y de las principales ciudades de la República” se derivó de la participación del país en la Pan-American Conference of Hygiene, reunida en Washington el mismo ańo, a la que asistió como delegado Pablo García Medina ${ }^{53}$, quien había publicado en 1907 el Tratado elemental de higiene y nociones de fisiología para la enseñanza de estas materias en escuelas y colegios de Colombia. En efecto, el tratado de García guardaba relación con la política sanitaria que él ayudó a fundamentar, la cual contemplaba la enseńanza gradual de tres ámbitos de la higiene en la educación oficial: la individual o privada, la urbana o pública y la social ${ }^{54}$.

51 Tomás Cadavid y David Velásquez, Informe de la Casa de Menores y Escuela de Trabajo San José (Medellín: Imprenta Oficial, 1921), 48-49. Biblioteca Central U. de A, Patrimonio documental, Sala Antioquia.

52 Pablo Llinás y Manuel Ortiz, "Hospicio". Informe que rinde la Junta General de Beneficencia de Cundinamarca a la Asamblea Departamental (Bogotá: Imprenta de La Luz, 1922), XLV. Archivo Central de la Beneficencia de Cundinamarca.

53 Médico fisiólogo de la Universidad Nacional de Colombia, precursor de la higiene y la salud pública en el país, director general de Higiene, Salubridad y Asistencia Públicas, profesor de la Facultad de Medicina de Bogotá.

54 Pablo García, Tratado elemental de higiene y nociones de fisiología para la enseñanza de estas materias en escuelas y colegios de Colombia (Bogotá: Arboleda y Valencia, 1915). 
Se destaca uno de los postulados de la política sanitaria que buscaba establecer la adopción de "sistemas eugénicos en resguardo de la vitalidad de la raza, que proteja y eduque a la madre y al niño, que emprenda una enérgica campaña en favor de la infancia, en el triple concepto de la higiene del medio, de la eugenesia y de la homicultura, y que ejercite, en fin, en todos los campos de la medicina social, una acción perseverante y enérgica" 55 . Estos preceptos confirman la convergencia entre eugenesia, puericultura e higiene mental, sustentada en los apartados anteriores y en adelante.

Esta hibridación científica, también se constata en la Tercera Conferencia Panamericana de Eugenesia y Homicultura, realizada en Bogotá en 1938, bajo la coordinación del médico Jorge Bejarano, Director Nacional de Sanidad, evento en el que se acordó que mejor sesionaría como parte del programa de la Décima Conferencia Sanitaria Panamericana. Finalmente, se consideró que sus objetivos eran más afines a los del Congreso Panamericano del Niño, organizado desde 1915, que a los de la Conferencia Sanitaria; por lo tanto, resolvieron celebrar sus próximas convocatorias conjuntamente con los de dicho Congreso.

No obstante, según Nancy Stepan, este cambio, más que retornar a la puericultura como un campo de la eugenesia, implicó una postura de distanciamiento frente al desprestigio de dicho término ${ }^{56}$, extremado por la política médica nazi para el exterminio de la "vida indigna de ser vivida" (Lebensunwertes Leben), que ejecutó el proyecto de "eutanasia compasiva" a grupos de niños anormales, locos e individuos en general que degeneraban la raza.

Entre las décadas del veinte y treinta del siglo XX, no se encuentran indicios de que en el país haya existido como tal un movimiento o liga de higiene mental, aunque las fuentes muestran que a partir de 1932 surgió una propuesta institucional de creación de un Centro de Higiene Física y

55 República de Colombia, Política Sanitaria. Exposición de motivos y Ley 12 de 1926, sobre enseñanza de la higiene, saneamiento de los puertos marítimos, fluviales y terrestres y de las principales ciudades de la República (Bogotá: Imprenta Nacional, 1926).

56 Nancy Stepan, The hour of eugenics: Race, Gender, and Nation in Latin America (New York: Cornell University Press, 1991), 194. 
Mental en la ciudad de Medellín, por parte de Eduardo Vasco ${ }^{57}$, primer psiquiatra infantil que ejerció en Antioquia, quien publicó su popular libro Breviario de la madre sobre puericultura, entendida como eugenesia.

En el país, las publicaciones sobre esta materia en revistas científicas empezaron a circular a partir de la década del treinta con los aportes de Vasco en los Anales de la Academia de Medicina de Medellín, los cuales fueron reunidos en su libro Temas de higiene mental, educación y engenesia publicado en 1948.

La mayoría de contribuciones se publicaron a finales de la década del cuarenta y durante la del cincuenta, por parte de psiquiatras, pediatras y pedagogos, en las revistas: Asociación Colombiana de Pediatría y Puericultura; Anales Neuropsiquiátricos en Bogotá, así como Antioquia Médica y Boletín Clínico de Medellín. De igual modo, se destaca que están enfocadas a la infancia y prevalece la puericultura como una rama de la eugenesia.

Según Abel Martínez, en el país la primera referencia sobre eugenesia, específicamente, fue publicada en 1940 por Héctor Pedraza en el artículo titulado "Eugenesia" en la Revista de Higiene de Bogotá, quien además publicó ese año con Rubén Gamboa el libro Higiene integral y alimentación del nińo, que empieza el primer apartado con el título de "Eugenesia"s8, en el que se refiere a los planteamientos expuestos de esta teoría para fundamentar una política que fuera implementada en el territorio nacional. Por tal razón, de acuerdo con Martínez, el término no surgió en el debate de la degeneración iniciado en la década del veinte por Jiménez López, como quedó fijado en el imaginario científico del país, sino 20 años después.

57 Médico de la Universidad de Antioquia, estudió en Ginebra y Bruselas, discípulo de Édouard Claparède, donde cursó el diplomado de Consejero de Orientación Profesional y Psicotécnica del Instituto de Altos Estudios de Bruselas, entre 1929 y 1930. Estuvo de asistente en la Clínica de Niños difíciles en la que conoció personalmente a Ovide Decroly. Estos autores fueron precursores de la Ligue internationale pour liéducation nouvelle, fundada en 1921, quienes influyeron en la pedagogía activa y la higiene mental en las casas de menores en Colombia, como se verá, fue apropiada inicialmente por Tomás Cadavid desde la década del veinte.

58 Abel Martínez, "La mayor controversia científica de la intelectualidad colombiana. Miguel Jiménez López y la Degeneración de la Raza (1913-1935)" (tesis de Doctorado en Historia. Universidad Pedagógica y Tecnológica de Colombia - UPTC, 2015), 71. Nota: También se publicó el mismo año el artículo de Emiliano Vicaria, "Eugenesia y Escolaridad", Revista de Medicina y Cirugía, , Vol. 7, No. 1, enero (1940): 155-163. 
En 1953, la Sociedad de Psicopatología, Neurología y Medicina Legal de Colombia, fundada en 1944, organizó el Primer Congreso Nacional de Neurología, Psiquiatría y Medicina Forense, en el que recomendó al Ministro de Higiene, "proceder a fundar en Bogotá un instituto de higiene mental como medio eficaz de lucha contra las enfermedades mentales, y como recurso de gran valor en la prevención del delito y de la conducta irregular del niño". Asimismo, resolvió declarar constituida la Liga de Higiene Mental de Colombia, "una vez constituida la Liga se procederá a convocar el Primer Congreso de Higiene Mental de Colombia, con representantes del cuerpo médico de las sociedades de Neuropatología y Psiquiatría, de la justicia penal, de la Iglesia, de los juzgados de menores, de los pedagogos, psicólogos"59.

$\mathrm{Al}$ año siguiente, 1954, la segunda versión del Congreso, programó un simposio sobre higiene mental y entre sus consideraciones insistieron al Ministerio de Salud Pública crear un Secretariado de Higiene Mental con sede en Bogotá, asimismo la creación de un Departamento de Higiene Mental y Social ${ }^{60}$. Sin embargo, tanto las iniciativas del primer congreso como del segundo no lograron concretarse.

De igual forma, en 1955, el V Congreso Médico Social de la Confederación Médica Panamericana, reunido en Bogotá, incluyó entre los temas principales el de la higiene mental, en el que presentó un variado grupo de trabajos de Colombia en este campo. Asimismo, el Congreso Médico Nacional, en 1959, reunido en la capital, programó como uno de sus temas principales el de la higiene mental.

En 1956, la Beneficencia de Cundinamarca creó el Instituto de Higiene Mental, bajo la dirección de Julián Córdoba Carvajal ${ }^{61}$-quien fuera uno

59 Sociedad de Psicopatología, Neurología y Medicina Legal de Colombia, "Votos aprobados por el Primer Congreso Nacional de Neurología, Psiquiatría y Medicina Forense en su sección de clausura del 1 de marzo de 1953", Revista de Medicina Legal de Colombia, No. 13, junio-diciembre (1953): 82 .

60 Sociedad de Psicopatología, Neurología y Medicina Legal de Colombia, "Documentos relacionados con el Segundo Congreso Nacional de Neurología, Psiquiatría y Medicina Legal”, Antioquia Médica Vol. 5 No. 1, febrero (1953): 3.

61 Se destaca la presentación de una ponencia del director del IHM en el Congreso Panamericano de Higiene Mental en Brasil en 1935. 
de los fundadores de la Asociación Psicoanalítica Colombiana en 1962- el cual estaba conformado por los directores científicos de cuatro instituciones de asistencia social de la entidad, que si bien interactuaban con los asilos psiquiátricos, concentró sus acciones en la población infantil de la Escuela de Orientación Femenina, el Hospicio Campestre de Sibaté, y el Instituto San José, así como del adulto mayor en la Dirección de ancianatos.

Posteriormente, las fuentes ubican la fundación de una Liga Colombiana de Higiene Mental en la década del sesenta, a partir de la creación del Centro Piloto de HM, adscrito a la Secretaría de Educación de Bogotá, por Acuerdo 110 de 1960 con tres fines: 1) Preparación del magisterio en pedagogía, 2) Investigación de los problemas psicopedagógicos, 3) Rehabilitación y tratamiento de los nińos con problemas emocionales de conducta y del aprendizaje $e^{62}$.

Ya en la prensa se había hecho un llamado en 1959, para que "psicoanalistas" graduados recientemente de la Universidad Nacional, asesoraran a los profesores de básica primaria para plantear ante las autoridades educativas la enseńanza de la higiene mental y la educación sexual de la niñez ${ }^{63}$.

La Liga y el CPHM estuvieron bajo la dirección de Guillermo Sánchez Sanín ${ }^{64}$, entre 1960 y 1972. En compañía de Guillermo Correal Sanín, psiquiatra infantil y profesor de la práctica de HM de la Facultad de Psicología, instalaron la Liga durante la primera Semana de HM, realizada en Bogotá, el 27 de noviembre de 1961, con la participación de instancias como: Secretaría de Higiene del Distrito, Secretaría de Educación, Secretaría de Asistencia y Protección, Secretaría de Tránsito, Dirección de Educación Sanitaria del Servicio Cooperativo Interamericano de Salud Pública ${ }^{65}$.

62 Guillermo Sánchez, "Readaptación escolar. La higiene mental en la educación. Labores desarrolladas por el Centro Piloto de Higiene del Distrito Especial”, El Tiempo, Bogotá, 8 de noviembre de 1962.

63 El Tiempo, "Invitación a los sicólogos", El correo de El Tiempo, Bogotá ,9 mayo de 1959.

64 Médico y psicoanalista, se desempeñó como Psicoterapeuta de Grupo en el Ministerio de Justicia División de Menores, Bogotá de 1963 a 1965; Miembro del Comité Nacional de Salud Mental de 1967 a 1972; Miembro Fundador en 1961 de la Asociación Colombiana de Psiquiatría. Tomado de: Academia Nacional de Medicina de Colombia. Recuperado 31/08/2015. En: http://www.anmdecolombia.net/attachments/article/384/GUILLERMO\%20S\%C3\%81NCHEZ\%20MEDINA.pdf

65 El Tiempo “Semana de Higiene Mental inicia el próximo lunes”, Bogotá, 24 de noviembre de 1961. 
En este evento sesionaron siete mesas temáticas: la primera sobre la "Liga de HM", seguida de "La HM y la actitud de la familia ante el escolar", "El escolar y su rendimiento", "Pubertad y escuela", "Escolaridad y delincuencia", "Estímulo y castigo escolar", "HM y religión".

En un informe presentado por el director Sánchez, destacó que uno de los avances más sobresalientes del Centro Piloto de HM, fue la introducción de la palabra "mental" como término técnico dentro de la educación, puesto que precisaba que la mente del niño, su psiquismo, personalidad, no funcionaban aisladas en el proceso del aprendizaje, en tanto "La memoria y los conocimientos acumulados por esta son una parte, pero la otra es el cuerpo, su capacidad y función física, biológica, que tienen una mente, la cual siente, desea y decide. Todas estas partes hay que verlas cuando educamos y pensamos en salud mental" 66 .

Sánchez aproximó el concepto de higiene mental al de salud, definido por la OMS en 1948, entendido como "un estado de completo bienestar físico, mental y social, no solamente la ausencia de enfermedad o dolencia”, por lo tanto afirmaba que "Cuando hablamos de 'felicidad', paz, tranquilidad de la mente o satisfacciones, hablamos, querámoslo o no de salud mental; ella tiene que ver con todo... en el hogar, la escuela, el trabajo, la iglesia y en todas las distracciones" 67 .

En otro artículo de 1962, el CPHM reportó que el $36 \%$ de los niños asistentes a las escuelas del Distrito padecía de trastornos mentales, razón por la que hacían un llamado para adelantar "una permanente y coordinada campaña en defensa de la salud de la infancia y de su protección genérica. Factores de los cuales depende, en grado primordial, el mejoramiento humano del país y su propia supervivencia como colectividad civilizada" ${ }^{68}$.

En cuanto a la terapéutica, disponía de un consultorio para la atención infantil, dependencia en la que se insistía en que "no podemos seguir aplicando métodos anti-psicopedagógicos en la educación y si lo hacemos,

66 Guillermo Sánchez, Readaptación escolar, p. 5.

67 Guillermo Sánchez, Readaptación escolar, p. 5.

68 El Tiempo, "La Salud Infantil", Bogotá, octubre 29 de 1962. 
tendremos en las futuras generaciones, hombres en donde la rebeldía se expresará en la violencia y su creatividad potencial anulada o puesta al servicio del pillaje, delincuencia y destrucción" ${ }^{69}$.

A este centro conducían, principalmente, niños díscolos que recogía la policía en las calles, así como oligofrénicos, epilépticos, psicóticos, neuróticos y con problemas de conducta, enviados por centros de salud, consultorios médicos, juzgados de menores, al igual que los conducidos por sus mismos padres o familiares.

En 1966, se propuso la construcción de un "Edificio de Conducta Infantil", que incluía una Clínica de Conducta, con la doble finalidad de servir de centro de observación para los niños "vagos" y de clínica de tratamiento psiquiátrico para los "menores" que presentaran trastornos mentales; asimismo asistía a los jardines infantiles distritales. Este centro realizaría "una labor de higiene mental más amplia y en general preparar mejor a la familia en todos los aspectos del cuidado de los niños"70.

La sección de Educación y Divulgación Científica del Centro impartía cursos y conferencias a grupos de madres, padres, enfermeras, maestros, asistentes y auxiliares sociales; asimismo, presentaba artículos sobre HM en los diarios de mayor circulación del país, como en este caso publicados en El Tiempo.

Se destaca la columna "Comentarios Médicos" de Jorge Bejarano (editada desde 1940 hasta 1965, que alcanzó 256 artículos en 26 años) $)^{71}$, en la que informó de su participación en la Comisión organizadora de la Primera Conferencia Nacional sobre Alcoholismo, convocada por el Departamento

69 Guillermo Sánchez, Readaptación escolar,. p. 5.

70 El Tiempo, "Otros catorce jardines infantiles para Bogotá", 19 junio de 1966.

71 En su columna "mezclaba cuestiones sanitarias con asuntos políticos, sobre la base de un lenguaje sencillo pero técnico, dirigido a diversos destinatarios, principalmente, miembros de las élites...”. Su publicación fue motivada por "la necesidad que manifestaron los lectores de tener mayores conocimientos en temas de higiene, en el contexto de una epidemia de parálisis infantil que afectaba varias regiones de Colombia”. Los macrotemas fueron agrupados en: Asuntos de gobierno (195 fuentes), alcohol (95), educación (93), asuntos campesinos (90), infancia (86) y alimentación (79). Ver: Rodrigo Ospina, "Jorge Bejarano: un intelectual orgánico del Partido Liberal, 1888-1966" (tesis de Maestría en Historia, Universidad Nacional de Colombia, Bogotá, 2012), 142. 
de Higiene Mental del Ministerio de Salud Pública del Perú y la Comisión organizadora del Congreso Latinoamericano de Salud Mental en Lima en 1958, en la que aseveró que en Colombia se desconocía la propensión heredo-degenerativa que pesaba sobre el aumento de la delincuencia infantil y con problemas de conducta, puesto que más de la mitad de estos nińos eran hijos de padres alcohólicos, asimismo, ocasionaba improductividad laboral y el aumento de internos por alcoholismo en cárceles y manicomios $^{72}$; como se sustentó, eran postulados propios del degeneracionismo, que aún pervivían en la década del sesenta en el país.

En la "Sección R/ Su salud” del periódico El Tiempo, se traducían las columnas “¿Cómo mantenerse sano?” (How to Keep Well?) y "Mantener su niño sano" (Keep Your Child Well) escritas desde 1959 por Theodore R. Van Dellen, médico editor de la sección de salud del Chicago Tribune, en las que se definió la HM como "habilidad para ajustarse a los cambios constantes que se presentan en las situaciones de la vida. Elasticidad emocional y gran habilidad para tomar decisiones"73.

En 1960, se dispuso la creación del Comité Colombiano para la Salud Mental, el cual en mayo de 1961 dio origen a la Liga Colombiana para la Salud Mental, entidad privada, afiliada a la Federación Mundial para la Salud Mental, presidida por Guillermo Correal Sanín; sin embargo, según Rosselli, las actividades de la Liga fueron muy escasas y, por tanto, tuvieron poco impacto en la población ${ }^{74}$.

A estas instancias le siguió la fundación de la Sección de Salud Mental del Ministerio de Salud, mediante el Artículo 33 del Decreto 3224 de 1963. Este fue el punto de partida para la aprobación de la Resolución 000679 que estableció las "Normas y reglamentación interna para unidades psiquiátricas en hospitales generales" y la creación del Consejo Nacional de Salud Mental, por Decreto 3060 de 1965, el cual sentó las bases para in-

72 Jorge Bejarano, "Comentarios Médicos”, El Tiempo, Bogota, 3 de noviembre de 1957.

11473 Theodore Van Dellen, "Higiene mental", Sección R/Su salud, El Tiempo, Bogotá, 22 de julio de 1961.

74 Humberto Rosselli, Historia de la Psiquiatría en Colombia, T. 2 (Bogotá: Editorial Horizonte, 1968), 648. 
cluir por primera vez un Programa de Salud Mental ${ }^{75}$ en el Plan Nacional de Salud 1968-1977. Es así como, este contexto histórico y marco normativo marcaron el despliegue de la higiene mental a la salud mental en Colombia.

\section{Conclusiones}

El predominio de la propensión hereditaria como determinante de la degeneración, por sobre los factores ambientales, fue la base empírica de la eugenesia de Francis Galton, quien estableció el perfeccionamiento biomédico de la raza o especie humana, extendido a tres campos: la higiene (física, mental y moral), la puericultura o cuidado materno-infantil, y la homicultura para "labrar el cuerpo y cosechar al hombre"

La higiene mental (HM) constituyó una solución técnica para prevenir y remediar la decadencia de la raza a la que se atribuía el atraso civilizatorio en que se encontraba sumido el país, trasfondo histórico que caracterizó el surgimiento de la incorporación del cuidado psíquico en la agenda de la salud pública en Colombia y Latinoamérica.

Se destaca que las ligas de HM tuvieron en común su origen y funcionamiento en las asociaciones de neurología y psiquiatría, asimismo una estrecha relación de ambas con las sociedades y agencias de eugenesia, entre las décadas de 1920 y 1930, según el registro de Sociedades e instituciones científicas para la América Latina de la Oficina Sanitaria Panamericana (Brasil, Chile, Perú, Uruguay, España, Cuba, Argentina, México) ${ }^{77}$. De igual modo, todas contaban con una sección de puericultura y, al mismo tiempo, las sociedades de esta especialidad y las de pediatría, junto con las ligas de HM, incluyeron una sección de eugenesia.

La "era de la infancia", denominación con la cual cierta historiografía ca-

75 Ministerio de Salud Pública, "Plan Nacional de Salud 1968-1977” (Bogotá: Imprenta P.E.S, 1967).

76 Zandra Pedraza, En cuerpo y alma., 211.

77 Oficina Sanitaria Panamericana, "Sociedades e instituciones científicas de la América Latina", Washington D.C., diciembre de 1939, Publicaciones mimeografiadas; 84 , 38, repositorio de la OPS/ OMS: http://iris.paho.org/xmlui/handle/123456789/1092 
racteriza al siglo XX, y en particular su primera mitad, articuló discursos, saberes y prácticas, según el influjo de las teorías degeneracionistas. Con base en estas se extendió la higiene física, mental y moral a tres ámbitos: el individual o privado, el urbano o público y el social. Esta profilaxis combinada fue un dispositivo utilizado para la prevención e intervención de los factores predisponentes (o hereditarios) y determinantes (o extrínsecos) que ocasionaban el supuesto retraso de la especie humana en que se hallaban sumidas las naciones en tránsito hacia la modernidad.

En cuanto a la articulación de los "saberes psi", de acuerdo con Ana Talak, es preciso considerar que la producción de discursos académicos que constituyeron la psicología general, dependió de la apropiación de diferentes disciplinas y profesiones ya conformadas, como son la medicina y psiquiatría, criminología, educación, entre otras ${ }^{78}$.

La psicotecnia, como base científica de la higiene mental, implementó la medición de capacidades mentales y corporales, a partir de la orientación de la "psicología experimental" que se usó muchas veces como sinónimo de "psicología científica” y, a su vez, asimilada en la pedagogía experimental o psicopedagogía, en tanto concentró su trabajo de campo en la población de las instituciones de protección infantil y corrección de menores.

\section{Bibliografía}

\section{Patrimonio documental}

Archivo Central de la Beneficencia de Cundinamarca, Fondo Sindicatura General, Secciones 10A-10B.

Alcerro, Ramón. Informe acerca de las actividades de la Segunda Reunión de la Asociación Psiquiátrica de América Latina y del IV Congreso Mundial de Salud Mental. Revista Médica Hondureña, No. 20, (1952): 80-88.

Bejarano, Jorge. "Comentarios Médicos". El Tiempo, Bogotá, 3 de noviembre de 1957.

78 Ana M. Talak, "Eugenesia e higiene mental: usos de la psicología en la Argentina, 1900-1940”, en Darwinismo social y eugenesia en el mundo latino, eds. Marisa Miranda y Gustavo Vallejo (Buenos Aires: Siglo XXI, 2005), 563-599. 
Borda, Alberto. "Higiene escolar y edificios para las escuelas". En Segundo Congreso Médico de Colombia. Bogotá: Escuela Tipográfica Salesiana, 1913.

Cadavid, Tomás y David Velásquez. Informe de la Casa de Menores y Escuela de Trabajo San José. Medellín: Imprenta Oficial, 1921, 48-49. Biblioteca Central U. de A., Patrimonio documental, Sala Antioquia. Castro, Alfonso. Degeneración colombiana. Medellín: Lit. e Imp. J.L. Arango, 1920.

Cuervo, Luis. "Quinto Congreso Médico Latinoamericano. Informe de la Delegación Colombiana”. Revista Médica de Bogotá Año 32, No. 380, febrero (1914).

Cuervo, Luis. “Medicina Social”. Revista Médica de Bogotá, Vol. 35, No. 418, abril, (1917): 204-207.

De Greiff, Carlos. "Mens sana in corpore sano". Anales de la Academia de Medicina de Medellín, Año XII Tomo 12, $\mathrm{N}^{\circ} 1$ y 2, agosto 30 (1903).

De Greiff, Carlos. Segunda conferencia sobre higiene, dictada en la Agrupación Central número $1^{\circ}$. En Instrucción Pública Antioqueña, Medellín, Colombia, Año 1, No. 5-6, julio, (1905): 195.

De Greiff, Carlos. Conferencias de Higiene en las Escuelas de Medellín. Medellín: Tipografía del Comercio, 1905. Sala Patrimonial Universidad Eafit, Medellín.

De Greiff, Carlos. "Decadencia Mental”. Anales de la Academia de Medicina de Medellín, Vol. 11. No. 1 y 2, agosto (1899): 48-52.

El Tiempo. Listo el temario de la reunión de directores de sanidad, 9 de marzo de 1940.

El Tiempo. "Invitación a los sicólogos" El correo de El Tiempo, Bogotá, 9 de mayo de 1959.

El Tiempo. "Semana de Higiene Mental inicia el próximo lunes". Bogotá, 24 de noviembre de 1961.

El Tiempo. "La Salud Infantil”. Bogotá, 29 de octubre de 1962.

El Tiempo. "Otros catorce jardines infantiles para Bogotá”. Bogotá, 19 de junio de 1966.

García, Pablo. Tratado elemental de higiene y nociones de fisiología para la enseñanza de estas materias en escuelas y colegios de Colombia. Bogotá: Arboleda y Valencia, 1915. 
Jiménez, Miguel, Luis López de Mesa, Jorge Bejarano, et al. Los problemas de la raza en Colombia, segundo volumen. Bogotá: Biblioteca de Cultura, 1920.

López de Mesa, Luis. "Higiene mental. Plan quinquenal del Ministerio de Educación Nacional”. Anales Neuropsiquiátricos, Vol. XI, (1957): 17. Escritos sobre medicina (Serie documental), folios 1-6, Archivos personales. Patrimonio documental U. de A.

Lyman, Ray. "Un mensaje de higiene social”. Jour. Sor. Hyg., 127, marzo (1937). Reproducido por la Oficina Sanitaria Panamericana, 1937. Llinás, Pablo y Manuel Ortiz. "Hospicio”. Informe que rinde la Junta General de Beneficencia de Cundinamarca a la Asamblea Departamental. Bogotá: Imprenta de La Luz, 1922. Archivo Central de la Beneficencia de Cundinamarca.

Ministerio de Salud Pública. Plan Nacional de Salud 1968-1977. Bogotá: Imprenta P.E.S, 1967.

Organización Mundial de la Salud. Informe de la primera reunión del Comité de Expertos en Higiene Mental, Ginebra, Oficina Sanitaria Panamericana, Washington, Serie de Informes Técnicos, 1949, No. 31, p. 7.

Organización Mundial de la Salud. Informe de la segunda reunión del Comité de Expertos en Higiene Mental, Ginebra, Oficina Sanitaria Panamericana, Washington, Serie de Informes Técnicos, 1950, No. 31, p. 2-3.

Organización Panamericana de la Salud. IV Conferencia Panamericana de Directores Nacionales de Sanidad celebrada en Washington, D.C., abril 30-mayo 8, 1940, Acta Final. Boletín de la Oficina Sanitaria Panamericana, Año 19, junio de 1940 No. 6, p. 1240.

Oficina Sanitaria Panamericana. Sociedades e instituciones científicas de la América Latina. Washington D.C. diciembre de 1939. Publicaciones mimeografiadas; 84, p. 38. Repositorio de la OPS/OMS: http://iris. paho.org/xmlui/handle/123456789/1092

Sociedad de Psicopatología, Neurología y Medicina Legal de Colombia. "Votos aprobados por el Primer Congreso Nacional de Neurología, Psiquiatría y Medicina Forense en su sección de clausura del $1^{\circ}$ de marzo de 1953". Revista de Medicina Legal de Colombia, Vols. 6970, No. 13, junio-diciembre de 1953. 
Sociedad de Psicopatología, Neurología y Medicina Legal de Colombia. "Documentos relacionados con el Segundo Congreso Nacional de Neurología, Psiquiatría y Medicina Legal”. Antioquia Médica, Vol. 1, No. 5, febrero (1953).

Van Dellen, Theodore. "Higiene mental”. Sección R/Su salud. El Tiempo, Bogotá, 22 de julio de 1961.

Vasco, Eduardo. Breviario de la madre. Medellín: Bedout, 1934. Biblioteca Central U. de A., Sala de Patrimonio Documental.

Vasco, Eduardo. Temas de higiene mental, educación y eugenesia. Medellín: Bedout, 1948.

Vernaza, José I. "Higiene escolar". (Tesis Facultad de Medicina y Ciencias Naturales, Universidad Nacional de Colombia. Bogotá: Editores Arboleda y Valencia, 1912).

\section{Bibliografía}

Academia Nacional de Medicina de Colombia. Guillermo Sánchez Medina. Recuperado 31/08/2015. En: http://www.anmdecolombia. net/attachments/article/384/GUILLERMO\%20S\%C3\%81NCHEZ\%20MEDINA.pdf

Anderson, Victor "The Organization and Operation of Child Guidance Clinics". The Public Health Journal, Vol. 16, No. 8, August (1925): 371-378. Recuperado 29/05/2015. Disponible en: https://www.jstor.org/stable/41973349?seq=1\#page_scan_tab_contents

Campos, Ricardo. De la higiene del aislamiento a la higiene de la libertad. La reforma de la institución manicomial en Francia (1860-1940). Frenia, Vol. 1, No. 1, (2001): 37-64.

Dagfal, Alejandro. "El pasaje de la higiene mental a la salud mental en la Argentina, 1920-1960. El caso de Enrique Pichon-Rivière”. Trashumante. Rev. Amer. Hit. Soc. No. 5 (2015): 10-36.

Foucault, Michel. Las palabras y las cosas. México: Siglo XXI, 1982.

George Rosen, Locura y sociedad, sociología histórica de la enfermedad mental. Madrid: Alianza, 1974.

Grob, Gerald. “The Mental Hygiene Movement”. En Mental Illnes and American Society, 1875-1940 (cap. 6). Princeton: Princeton University Press, 1987. Traducción: María Laura Moukarzel. Revisión: Hernán Scholten y Agustín Kripper.

Lemkau, Paul V. Higiene mental. México: Fondo de Cultura Económica, 1963. 
Lopera, Juan D. "Una historia del concepto técnico de salud mental: entre ciencia e ideología”. En Salud y Salud Pública. Aproximaciones históricas y epistemológicas, editado por Álvaro Casas y Jana Congote, Facultad Nacional de Salud Pública, UdeA - Hombre Nuevo Editores, 2013.

Lopera, Juan D. "Sabiduría práctica y salud psíquica”. (Tesis de Doctorado en Ciencias Sociales, Universidad de Antioquia, Medellín, 2014). Publicada en libro: Bogotá: Editorial San Pablo - Fondo Editorial Universidad Eafit, 2016.

Martínez, Abel. "La mayor controversia científica de la intelectualidad colombiana. Miguel Jiménez López y la Degeneración de la Raza (1913-1935)". (Tesis de Doctorado en Historia, Universidad Pedagógica y Tecnológica de Colombia - UPTC, 2015).

Ospina, Rodrigo. "Jorge Bejarano: un intelectual orgánico del Partido Liberal, 1888-1966". (Tesis de Maestría en Historia, Universidad Nacional de Colombia, Bogotá, 2012), 142.

Pedraza, Zandra. En cuerpo y alma. Visiones del progreso y de la felicidad. Educación, cuerpo y orden social en Colombia (1830-1990). Bogotá: Universidad de los Andes, 2011.

República de Colombia. Ley 30 de 1886 del Consejo Nacional Legislativo: que crea juntas de higiene en la capital de la república y las de los departamentos o ciudades principales. En: Revista de Instrucción Pública, Vol. 19, No. 07, julio de 1906.

República de Colombia. Política Sanitaria. Exposición de motivos y Ley 12 de 1926, sobre enseńanza de la higiene, saneamiento de los puertos marítimos, fluviales y terrestres y de las principales ciudades de la República. Bogotá: Imprenta Nacional, 1926.

Ríos, Andrés ¿Cómo prevenir la locura? Psiquiatría e higiene mental en México, 1934-1950. México D.F.: UNAM - Siglo XXI, 2016.

Rosen, George. Locura y sociedad, sociología histórica de la enfermedad mental. Madrid: Alianza, 1974.

Rosen, George. De la policía médica a la medicina social. Ensayos sobre la historia de la atención en salud. México: Siglo XXI, 2005.

120 Rosselli, Humberto. Historia de la Psiquiatría en Colombia. T. 2. Bogotá: Editorial Horizonte, 1968.

Sánchez, Guillermo. "Readaptación escolar. La higiene mental en la edu- 
cación. Labores desarrolladas por el Centro Piloto de Higiene del Distrito Especial”. El Tiempo, Bogotá, 8 de noviembre de 1962.

Silva, José A. "Espacio, cuerpo y subjetividad en el Manicomio Departamental de Antioquia: 1875-1930”. (Tesis de Maestría en Historia, Universidad Nacional de Colombia, 2012).

Stepan, Nancy. The hour of eugenics: Race, Gender, and Nation in Latin America. New York: Cornell University Press, 1991.

Talak, Ana M. Eugenesia e higiene mental: usos de la psicología en la Argentina, 1900-1940. En Darwinismo social y eugenesia en el mundo latino, editado por Marisa Miranda y Gustavo Vallejo. Buenos Aires: Siglo XXI, 2005, 563-599.

Para citar este artículo: Gutiérrez Avendaño, Jairo. "Mens sana in corpore sano: incorporación de la higiene mental en la salud pública en la primera mitad del siglo XX en Colombia”, Historia Caribe Vol. XIV No. 34 (Enero-Junio 2019): 91-121. DOI: http://dx.doi.org/10.15648/ hc. 34.2019 .3 